\title{
ORIGINAL ARTICLE \\ Early versus late surgery for traumatic spinal cord injury: the results of a prospective Canadian cohort study
}

\author{
JR Wilson ${ }^{1}$, A Singh ${ }^{1}$, C Craven ${ }^{2}, \mathrm{MC} \mathrm{Verrier}^{3}, \mathrm{~B} \mathrm{Drew}^{4}, \mathrm{H} \mathrm{Ahn}{ }^{5}, \mathrm{M} \mathrm{Ford}^{6}$ and MG Fehlings ${ }^{1}$
}

Study design: A multicenter Canadian cohort study.

Objectives: The objective of this study is to evaluate the impact of early versus late surgical decompression on motor neurological recovery after traumatic spinal cord injury (SCI).

Setting: Canadian acute care and $\mathrm{SCl}$ rehabilitation facilities.

Methods: A prospective cohort study of patients within the Ontario Spinal Cord Injury Registry program was performed. We considered SCI patients with an admission American Spinal Injury Association (ASIA) Impairment Scale (AIS) grade of A through D, with magnetic resonance imaging-confirmed spinal cord compression. Grouped analysis was performed comparing the cohort of patients who received early surgery $(<24 \mathrm{~h}$ after $\mathrm{SCl})$ to those receiving delayed surgery $(\geqslant 24 \mathrm{~h}$ after $\mathrm{SCl})$. The primary outcome was the change in ASIA motor score (AMS) occurring between hospital admission and rehabilitation discharge.

Results: A total of $35(41.7 \%)$ patients underwent early surgery and $49(58.3 \%)$ underwent late surgery. At admission, there was a greater proportion of patients within the early surgery group with more severe AIS grade A injuries. Of the 55 patients with neurological exam available at rehabilitation discharge, a greater proportion had at least a two-grade AIS improvement in the early-surgery group $(P=0.01)$. The mean improvement in AMS at rehabilitation discharge was 20 points amongst early-surgery patients and 15 points amongst late-surgery patients $(P=0.46)$. In the analysis investigating AMS improvement, adjusted for preoperative status and neurological level, there was a positive effect estimate for early surgical therapy that was statistically significant $(P=0.01)$.

Conclusion: The results here add weight to the growing body of literature, which supports the principle of early intervention in the setting of spinal trauma and $\mathrm{SCl}$.

Spinal Cord (2012) 50, 840-843; doi:10.1038/sc.2012.59; published online 8 May 2012

Keywords: spinal cord injury; neuroprotection; surgery; neurological outcomes

\section{INTRODUCTION}

The incidence of traumatic spinal cord injury (SCI) in Canada ranges from approximately 23 to 52 per million per year, with an additional $20 \%$ dying before arrival at the hospital. ${ }^{1,2}$ Although the frequency of SCI is relatively low, it disproportionately affects the younger population, leading to devastating personal losses and imposing a significant economic burden on society as a whole. ${ }^{3}$ A recent Canadian study estimated that the average SCI patient is hospitalized for approximately 139 days in the first year after the injury, with average healthcare costs per individual approaching 120000 Canadian dollars for the same period. ${ }^{4}$ In spite of promising advances in preclinical research, a uniformly safe and effective therapy that has been definitively proven to reduce the degree of neurological impairment and improve clinical outcomes remains elusive. $^{5}$

After the initial trauma, or primary injury, neurological tissue destruction is potentiated by a cascade of deleterious pathobiological processes, collectively referred to as secondary injury mechanisms. ${ }^{6}$ Although little can be done from a therapeutic standpoint to correct damage sustained during the primary injury, by mitigating the evolution of secondary injury events, there is an opportunity to preserve remnant viable neurological tissue and hence optimize outcomes. There is compelling preclinical evidence to suggest that persistent spinal cord compression exacerbates ongoing secondary injury processes and that surgical decompression of the spinal cord is neuroprotective, leading to improved functional outcomes in animal studies. ${ }^{7,8}$ Furthermore, the strength of this neuroprotective effect seems to vary inversely with the time elapsed from injury to the decompression. ${ }^{9}$ In the clinical realm, evidence for surgical decompression after SCI remains conflicted. However, all recommendations made to date have lacked the support of a large systematic comparative analysis evaluating the relative efficacy of various surgical timing cutoff points.

In the current study, we have chosen a 24-h cutoff point to evaluate the effect of surgical timing on outcomes after SCI. We have completed a prospective cohort study to compare the relative effectiveness of early surgical decompression, performed before $24 \mathrm{~h}$ post injury, to late surgical decompression, performed at or after $24 \mathrm{~h}$

${ }^{1}$ Department of Surgery, Division of Neurosurgery and Spinal Program, University of Toronto, Toronto Western Hospital, Toronto, Ontario, Canada; ${ }^{2}$ Division of Physical Medicine and Rehabilitation, Toronto Rehabilitation Institute, University of Toronto, Toronto, Ontario, Canada: ${ }^{3}$ Division of Rehabilitation Sciences, University of Toronto, Toronto, Ontario, Canada; ${ }^{4}$ Department of Surgery, Division of Orthopedic Surgery, McMaster University, Hamilton, Ontario, Canada; ${ }^{5}$ Department of Surgery, Division of Orthopedic Surgery and Spinal Program, University of Toronto, St Michael's Hospital, Toronto, Ontario, Canada and ${ }^{6}$ Department of Surgery, Division of Orthopedic Surgery and Spinal Program, University of Toronto, Sunnybrook Hospital, Toronto, Ontario, Canada

Correspondence: Dr MG Fehlings, Department of Surgery, Division of Neurosurgery and Spinal Program, University of Toronto, Toronto Western Hospital, 399 Bathurst Street, Toronto, Ontario, Canada M5T 2S8.

E-mail: Michael.Fehlings@uhn.on.ca

Received 9 February 2012; revised 19 March 2012; accepted 30 March 2012; published online 8 May 2012 
post injury, with respect to the primary outcome of motor recovery at rehabilitation discharge.

\section{METHODS}

A Canadian multicenter prospective cohort study was performed involving acute-care facilities and rehabilitation units at the University of Toronto (Toronto, Ontario, Canada) and McMaster University (Hamilton, Ontario, Canada). ${ }^{10}$ During the 2-year period between January 2007 and January 2009, any patient presenting with traumatic SCI to one of the participating centers was assessed for study eligibility against a predetermined set of exclusion and inclusion criteria (Table 1). In general, any patient with SCI originating from a traumatic etiology, with neurological deficit and radiological evidence of spinal cord compression was enrolled. The presence or absence of spinal cord compression was determined on the basis of the admission magnetic resonance imaging scan, specifically by applying the measurements previously described by the Fehlings group to the mid-sagittal T2 sequence for each patient. ${ }^{11}$ Also, at acute-care admission, all patients underwent neurological assessment as per the International Standards of Neurological Classification for Spinal Cord Injury and in concordance with the recommendation of the American Spinal Injury Association (ASIA) by a trained physician, nurse or research assistant. ${ }^{12}$ Injury characteristics were then classified according to neurologic level of injury, ASIA motor score (AMS), ASIA sensory score and the overall ASIA Impairment Scale (AIS) grade (A-D). Additional clinical parameters collected at admission included patient age, gender and injury etiology.

Patients were separated into two cohorts, depending on the timing of surgical decompression relative to the injury. The early-surgery cohort consisted of patients who underwent surgery before $24 \mathrm{~h}$ post injury and the late surgery cohort consisted of patients who underwent surgery at or after $24 \mathrm{~h}$ post injury. Group assignment was primarily dependent upon patients' pre-hospital transport time, the time for diagnostic work-up and medical stabilization, as well as surgeon preference in the management of the individual patient. All patients received optimal medical support, which included permissive or induced hypertensive therapy for 1 week following the injury. ${ }^{13}$ Methylprednisolone was used as per the discretion of the treating team according to the recommendations of the Second National Acute Spinal Cord Injury Study. ${ }^{14}$ Regarding surgical details, all decisions, including choice of approach, extent of decompression and the use of spinal instrumentation, were made on a case-by-case basis by the attending orthopedic or neurosurgeon involved. Following acute-care discharge, all patients underwent an individualized rehabilitation protocol in a spinal cord rehabilitation unit, tailored to specific patient needs and injury characteristics.

Table 1 Inclusion and exclusion criteria

\begin{tabular}{|c|c|}
\hline Inclusion criteria & Exclusion criteria \\
\hline Age $>16$ & $\begin{array}{l}\text { Cognitive impairment preventing accurate } \\
\text { neurological assessment }\end{array}$ \\
\hline Initial AIS grade A-D & Penetrating injuries \\
\hline $\begin{array}{l}\text { Spinal cord compression confirmed } \\
\text { by MRI or CT myelography }\end{array}$ & Pregnant females \\
\hline \multirow[t]{3}{*}{$\begin{array}{l}\text { Patient or proxy willing to provide } \\
\text { consent for enrollment }\end{array}$} & $\begin{array}{l}\text { Pre-injury major neurological deficits or } \\
\text { disease (i.e., ischemic stroke, Parkinson's } \\
\text { disease) }\end{array}$ \\
\hline & $\begin{array}{l}\text { Life-threatening injuries that prevent early } \\
\text { decompression of the spinal cord }\end{array}$ \\
\hline & $\begin{array}{l}\text { Significant pre-morbid medical illness } \\
\text { (including, but not limited to): } \\
\text { myocardial infarction within } 3 \text { months } \\
\text { uncompensated heart failure } \\
\text { active systemic cancer } \\
\text { AIDS }\end{array}$ \\
\hline
\end{tabular}

Abbreviations: AIS, ASIA Impairment Scale; CT, computed tomography; MRI, magnetic resonance imaging.

\section{Follow-up and outcomes of interes}

All patients were followed from the time of acute-care admission until the time of rehabilitation discharge according to original group assignments. Follow-up neurological evaluations were completed at two time points: acute-care discharge and in-patient rehabilitation discharge. The primary outcome of interest was motor recovery at rehabilitation discharge, defined by the change in AMS occurring between acute hospital admission and rehabilitation discharge. As a secondary neurological outcome measure, we also evaluated the change in the AIS grade between admission and the two follow-up points for each patient. In addition to neurological parameters, hospital length-of-stay information was also recorded at the two follow-up points.

\section{Statistical analysis}

All analyses were performed using SAS version 9.2 (SAS Institute Inc., Cary, NC, USA). Descriptive statistics were used to characterize patient demographics and injury characteristics. Results of continuous outcome measures, such as change in AMS and ASIA sensory scores at follow-up, were compared between the cohorts, using the Student's $t$-test. Comparison of the AIS grade improvements was performed using the $\chi^{2}$-test. An adjusted analysis was also completed to account for the effects of confounding variables, using linear regression. For this analysis, the outcome variable was defined as the change in AMS from pre-op to rehabilitation discharge, whereas the predictor variable of interest was defined as surgical timing (early versus late). Other predictor variables, such as preoperative neurological status (complete versus incomplete), age, gender and neurological level of injury, were included as predictors initially, and then sequentially eliminated in a backwards fashion if the corresponding $P$-value was greater than 0.05 .

\section{Statement of ethics}

We certify that all applicable institutional and governmental regulations concerning the ethical use of human volunteers were followed during the course of this research

\section{RESULTS}

A total of 84 patients with traumatic SCI were enrolled over the course of the 2-year study period. Of these, $35(41.7 \%)$ underwent surgery before $24 \mathrm{~h}$ after injury and were considered the early-surgery cohort, whereas $49(58.3 \%)$ underwent surgery at or after $24 \mathrm{~h}$ post injury and were considered the late-surgery cohort. The mean time to surgery was $12.7( \pm 4.9)$ and $155.0 \mathrm{~h}( \pm 236.7)$ in the early and late groups, respectively $(P<0.01)$. Table 2 provides a listing of the demographic and injury characteristics of both the early- and late-surgery cohorts. In both groups, there were a preponderance of males with $29(77.6 \%)$ in the early cohort and $40(82.9 \%)$ in the late cohort $(P=0.55)$. Although there was a trend towards a younger mean age within the early group (41.7 years in the early group versus 47.9 years in the late group), this difference was not significant $(P=0.09)$. With respect to neurological exam findings at admission, the two groups differed significantly with the AIS grade A injuries most common in the early group and AIS grade $\mathrm{D}$ injuries most common in the late group $(P=0.01)$. The cervical region was the most common injury location with thoracic and lumbo-sacral injuries encountered less frequently in both groups. Motor vehicle accidents and falls caused the majority of injuries, with no significant differences in etiology between the early and late group.

\section{Pre-op to acute-care discharge}

Of the original 84 patients, $82(97.6 \%)$ had neurological follow-up information available at acute-care discharge. The average length of acute-care hospital stay in the total study group was $24.8( \pm 29.2)$ days. There was no difference in the average length of acute hospital stay between the early (24.9 days) and late (24.7 days) surgery groups $(P=0.97)$. From the time of preoperative assessment until acute hospital discharge, seven patients $(21.2 \%)$ in the early group 
Table 2 Preoperative patient characteristics

\begin{tabular}{lccc}
\hline & $\begin{array}{c}\text { Early surgery } \\
(\mathrm{N}=35)\end{array}$ & $\begin{array}{c}\text { Late surgery } \\
(\mathrm{N}=49)\end{array}$ & P-value \\
Characteristic & & & 0.01 \\
\hline Pre-op AIS grade & $18(51)$ & $15(31)$ & \\
AIS A (\%) & $6(17)$ & $3(6)$ & \\
AIS B (\%) & $5(14)$ & $6(12)$ & \\
AIS C (\%) & $6(17)$ & $25(51)$ & \\
AIS D (\%) & & & \\
& 41.6 & 47.9 & 0.09 \\
Age & $29(83)$ & $38(78)$ & 0.61 \\
Male (\%) & & & \\
& & & \\
Etiology & $13(37.1)$ & $10(20.4)$ & \\
Motor vehicle accident (\%) & $13(37.1)$ & $29(59.2)$ & \\
Fall (\%) & $1(2.9)$ & $3(6.1)$ & \\
Assault (\%) & $8(22.9)$ & $7(14.3)$ & \\
Other (\%) & & & \\
& & & \\
Neurological level of injury & $14(40)$ & $30(61.2)$ & \\
Cervical (\%) & $12(34.3)$ & $9(18.4)$ & \\
Thoracic (\%) & $9(25.7)$ & $10(20.4)$ & \\
Lumbo-sacral (\%) & & & \\
Received (\%) & $3(12)$ & $7(19.4)$ & 0.44 \\
Methylprednisolone & 25 & 36 & \\
\hline
\end{tabular}

Abbreviation: AIS, ASIA Impairment Scale.

and nine patients (18.4\%) in the late group experienced at least a onegrade AIS improvement $(P=0.47)$, and three patients $(9.1 \%)$ in the early group and one patient $(2.0 \%)$ in the late group experienced at least a two-grade AIS improvement $(P=0.15)$. Also at this time point, the mean AMS improvement in the early and late groups were 6.2 and 9.7 points, respectively $(P=0.18)$.

\section{Pre-op to rehabilitation discharge}

Of the original 84 patients, $55(65.4 \%)$ had follow-up neurological information available at rehabilitation discharge. The mean length of rehabilitation stay was 89.6 ( \pm 47.4$)$ days, with no difference in the average length stay between the early (102.9 days) and late ( 80.2 days) surgery groups $(P=0.10)$. From preoperative assessment until rehabilitation discharge, 9 patients $(40.9 \%)$ in the early group and 10 patients $(30.3 \%)$ in the late group improved at least one AIS grade $(P=0.42)$, and 6 patients $(27.2 \%)$ in the early group and 1 patient $(3.0 \%)$ in the late group improved at least two AIS grades $(P=0.01)$ (Table 3). In terms of motor recovery, the mean improvement in AMS at rehabilitation discharge was 19.5 amongst early-surgery patients and 15.4 amongst late-surgery patients $(P=0.46)$.

For the adjusted analysis investigating AMS recovery, after stepwise backwards elimination, only surgical timing, preoperative neurological status and neurological level of injury remained, revealing an effect estimate of 13.0 for early surgical therapy that was statistically significant $(P=0.01$; Table 4$)$.

\section{DISCUSSION}

We have performed a prospective multicenter cohort study to evaluate the influence of surgical timing on neurological outcome after traumatic SCI. In considering the primary predefined outcome measure, AMS at rehabilitation discharge, the unadjusted analysis revealed a non-significant difference favoring the completion of early
Table 3 AIS grade conversion from pre-op to rehab discharge

\begin{tabular}{lrrrrrr}
\hline Pre-op AlS grade & $A$ & $B$ & $C$ & $D$ & $E$ & Total \\
\hline (a) Late surgery group & & & & & & \\
A & 12 & 0 & 0 & 0 & 0 & 12 \\
B & 0 & 0 & 1 & 1 & 0 & 2 \\
C & 0 & 0 & 0 & 5 & 0 & 5 \\
D & 0 & 0 & 0 & 11 & 3 & 14 \\
Total & 12 & 0 & 1 & 17 & 3 & 33 \\
(b) Early surgery group & & & & & & \\
A & 6 & 0 & 5 & 0 & 0 & 11 \\
B & 0 & 2 & 1 & 1 & 0 & 4 \\
C & 0 & 0 & 1 & 2 & 0 & 3 \\
D & 0 & 0 & 0 & 4 & 0 & 4 \\
Total & 6 & 2 & 6 & 8 & 0 & 22 \\
\hline
\end{tabular}

Abbreviation: AIS, ASIA Impairment Scale.

Table 4 Results of multivariate analysis predicting the change in AMS at rehabilitation discharge

\begin{tabular}{lcc}
\hline Predictor & Parameter estimate & P-value \\
\hline Early versus late surgery & 13.0 & 0.01 \\
Complete versus incomplete & -15.0 & 0.01 \\
& & $<0.01$ \\
Neurological level & & - \\
Lumbo-sacral & -26.0 & - \\
Thoracic & -7.2 & \\
Cervical (ref) & 0 & \\
\hline
\end{tabular}

Abbreviation: AMS, ASIA motor score.

surgery. However, when considered in the context of the multivariate analysis, which adjusted for relevant confounding variables, early surgery was found to be a statistically significant predictor of motor recovery. Stated plainly, after controlling for the neurological level of injury and baseline neurological status, patients who underwent surgical decompression of the spinal cord before $24 \mathrm{~h}$ after injury experienced an additional 13 points in motor recovery as compared with patients who underwent surgery at or after $24 \mathrm{~h}$. When considering other secondary neurological outcomes, there was a significantly greater proportion of patients who improved at least two AIS grades at rehabilitation discharge in the early surgery cohort. Lastly, there was a greater proportion of patients in the early surgery group, who improved at least one AIS grade, but this difference was not statistically significant.

There is compelling biological rationale to support the findings observed in the current study. On the basis of the sizable body of preclinical literature that has arisen over the years, it is clear that persistent spinal cord compression represents a form of secondary injury that potentiates the degree of ongoing tissue destruction and ultimately worsens neurological outcomes. ${ }^{15,16}$ In response to this, a number of studies have arisen to evaluate the pathological and functional effects of decompressive surgery in the setting of animal SCI with persistent spinal cord compression. Summarizing the available preclinical evidence, in a recent systematic review, Furlan et al. ${ }^{17}$ reported that of the 19 studies that met inclusion/exclusion criteria, 11 demonstrated superior neurobehavioral recovery and diminished pathological tissue destruction amongst animals that underwent earlier decompression of the spinal cord. Although recognizing the inherent difficulties in extrapolating laboratory 
findings to human populations, this work has led to the clinical hypothesis that patients with SCI and persistent spinal cord compression will experience superior neurological outcomes if treated, as early as possible, with surgical decompression.

Translating the promising results for spinal cord decompression from the preclinical realm into clinical practice has proven challenging in light of the practical realities of trauma care. As with any neuroprotective therapy that aims to reduce secondary injury mechanisms, the earlier the treatment is instituted, the greater the potential for neurological tissue preservation. However, factors such as the time required for pre-hospital transport, medical stabilization and diagnostic investigation delay the delivery of definitive surgical care. From a feasibility standpoint, one Canadian study and one European study have demonstrated that between 25 and $50 \%$ of patients underwent surgery before $24 \mathrm{~h}$ post injury. ${ }^{18,19}$ From an efficacy standpoint, a recent systematic review of clinical studies concluded that decompressive surgery performed before $24 \mathrm{~h}$ resulted in superior clinical outcomes as compared with decompression performed after the 24-h cutoff. ${ }^{20}$ Several studies, including those of McKinley et al. ${ }^{21}$ and Vaccaro et al.,22 have evaluated the more practical 72-h cutoff and failed to find a difference between early and late surgery, with respect to neurological outcomes at follow-up. Hence, defining an optimal time cutoff for surgical decompression reflects the need for optimizing efficacy within the practical constraints of the real world. After evaluating the relevant body of literature, we felt that the 24-h cutoff represented the most promising time window during which surgical decompression had the potential to confer a neuro-protective effect.

For medical practitioners involved with the care of trauma patients, the current study brings to light several important points related to the acute management of patients with traumatic SCI. Given that the potential utility of surgical decompression is contingent upon our ability to diagnose spinal cord compression, this study reinforces the need for a spinal magnetic resonance imaging scan to be completed in the immediate post-trauma period for all individuals with a suspected SCI. This will allow for prompt triage of patients who may derive benefit from surgery. Second, given that we have demonstrated the time-dependent potential for improved motor recovery with surgery, emergency doctors and frontline trauma physicians should attempt to expedite the diagnostic process, and the subsequent referral to a spine surgeon, in the face of a suspected SCI. Lastly, this study highlights the need for adjustments within current trauma systems to allow for a streamlined path to the operating room for those eligible for decompressive surgery.

\section{Study limitations}

We recognize that there are differences between the two cohorts, most notably with respect to preoperative neurological status, with the majority of patient in the early-surgery group AIS grade A and the majority of patients in the late-surgery group AIS grade D. Given that recovery amongst AIS grade D patients has the potential to be capped because of ceiling effects, we cannot exclude the possibility that this may have influenced study results. We have attempted to account for baseline differences between the cohorts by performing a multivariate analysis, adjusting for potential confounding variables. Also, we did not prospectively collect neurological-level data at rehabilitation discharge, which would have been of interest.

\section{CONCLUSION}

We have shown that for patients with SCI and persistent spinal cord compression, surgical decompression performed before $24 \mathrm{~h}$ post injury has the potential to result in superior motor recovery in comparison with late surgery performed at or after $24 \mathrm{~h}$ post injury. Further analyses are needed to more accurately define which patients stand to benefit the most from early operative intervention.

\section{DATA ARCHIVING}

There were no data to deposit.

\section{CONFLICT OF INTEREST}

The authors declare no conflict of interest.

\section{ACKNOWLEDGEMENTS}

We would like to thank the Ontario Neurotrauma Foundation and the Ontario Ministry of Health and Long-term Care for funds received to establish the Ontario Spinal Cord Injury Registry.

1 Dryden D, Saunders L, Rowe B, May L, Yiannakoulias N, Svenson L et al. The epidemiology of traumatic spinal cord injury in Alberta, Canada. Can J Neurol Sci 2003; 30: 113-121.

2 Pickett W, Simpson K, Walker J, Brison R. Traumatic spinal cord injury in Ontario, Canada. J Trauma 2003; 55: 1070-1076.

3 Ackery A, Tator C, Krassioukov A. A global perspective on spinal cord injury epidemiology. J Neurotrauma 2004; 21: 1355-1370.

4 Dryden D, Saunders L, Jacobs P, Schopflocher D, Rowe B, May L et al. Direct health care costs after traumatic spinal cord injury. J Trauma 2005; 59: 443-449.

5 Hawryluk G, Roland J, Kwon B, Fehlings M. Protection and repair of the injured spinal cord: a review of completed, ongoing, and planned clinical trials for acute spinal cord injury. Neurosurg Focus 2008; 25: E14.

6 Fehlings $M$ and Sekhon L eds. Cellular, lonic and Biomolecular Mechanisms of the Injury Process. American Association of Neurologic Surgeons: Chicago, 2000.

7 Guha A, Tator C, Endrenyi L. Decompression of the spinal cord improves recovery after acute experimental spinal cord compression injury. Paraplegia 1987; 25: 324-339.

8 Carlson G, Minato Y, Okada A, Gorden C, Warden K, Barbeau J et al. Early timedependent decompression for spinal cord injury: Vascular mechanisms of recovery. J Neurotrauma 1997; 14: 951-962.

9 Dimar J, Glassman S, Raque G, Zhang Y, Shields C. The influence of spinal canal narrowing and timing of decompression on neurologic recovery after spinal cord contusion in a rat model. Spine 1999; 24: 1623-1633.

10 Verrier M, Ahn H, Craven C, Drew B, Fehlings M, Ford M. Ontario spinal cord injury informatics: informing practice, research and innovations. Ontario Neurotrauma Foundation 2009, Available from http://www.onf.org/documents/OSCIR.pdf.

11 Fehlings M, Rao S, Tator C, Skaf G, Arnold P, Benzel E et al. The optimal radiologic method for assessing spinal canal compromise and cord compression in patients with cervical spinal cord injury. Part 2: results of a multicenter study. Spine 1999; 24: 605-613.

12 American Spinal Injury Association ed. International Standards for Neurological Classification of Spinal Cord Injury. American Spinal Injury Association: Chicago, IL, 2000.

13 Hadley M, Walters B, Grabb P, Oyesiku N, Przybylski G, Resnick D et al. Blood pressure management after acute spinal cord injury. Neurosurgery 2002; 50: S58-S62.

14 Bracken M, Shepard M, Collins W, Holford T, Young W, Baskin D et al. A randomized, controlled trial of methylprednisolone or naloxone in the treatment of acute spinal-cord injury. Results of the Second National Acute Spinal Cord Injury Study. New Engl J Med 1990; 17: 1405-1411.

15 Tarlov I. Spinal cord compression studies. III. Time limits for recovery after gradual compression in dogs. AMA Arch Neurol Psychiatry 1954; 71: 588-597.

16 Carlson G, Gorden C, Oliff H, Pillai J, LaManna J. Sustained spinal cord compression: Part I: time-dependent effect on long-term pathophysiology. J Bone Joint Surg Am 2003; 85: 86-94.

17 Furlan J, Noonan V, Cadotte D, Fehlings M. Timing of decompressive surgery of spinal cord after traumatic spinal cord injury: an evidence-based examination of pre-clinical and clinical studies. J Neurotrauma 2009; 27: 1-29.

18 Tator C, Fehlings M, Thorpe K, Taylor W. Current use and timing of spinal surgery for management of acute spinal surgery for management of acute spinal cord injury in North America: results of a retrospective multicenter study. J Neurosurgery 1999; 91: 12-18.

19 Botel U, Glaser E, Niedeggen A. The surgical treatment of acute spinal paralysed patients. Spinal Cord 1997; 35: 420-428.

20 LaRosa G, Conti A, Cardali S, Cacciola F, Tomasello F. Does early decompression improve neurological outcome of spinal cord injured patients? Appraisal of the literature using a meta-analytical approach. Spinal Cord 2004; 42: 503-512.

21 McKinley W, Meade M, Kirshblum S, Barnard B. Outcomes of early surgical management versus late or no surgical intervention after acute spinal cord injury. Arch Phys Med Rehab 2004; 85: 1818-1825.

22 Vaccaro A, Daugherty R, Sheehan T, Dante S, Cotler J, Balderston R et al. Neurologic outcome of early versus late surgery for cervical spinal cord injury. Spine 1997; 22: $609-612$. 